RAIRO Operations Research

RAIRO Oper. Res. 38 (2004) 255-274

DOI: $10.1051 /$ ro: 2004023

\title{
ON CONSTRAINT QUALIFICATIONS IN DIRECTIONALLY DIFFERENTIABLE MULTIOBJECTIVE OPTIMIZATION PROBLEMS *
}

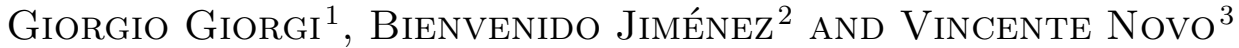 \\ Communicated by Jean Abadie
}

\begin{abstract}
We consider a multiobjective optimization problem with a feasible set defined by inequality and equality constraints such that all functions are, at least, Dini differentiable (in some cases, Hadamard differentiable and sometimes, quasiconvex). Several constraint qualifications are given in such a way that generalize both the qualifications introduced by Maeda and the classical ones, when the functions are differentiable. The relationships between them are analyzed. Finally, we give several Kuhn-Tucker type necessary conditions for a point to be Pareto minimum under the weaker constraint qualifications here proposed.
\end{abstract}

Keywords. Multiobjective optimization problems, constraint qualification, necessary conditions for Pareto minimum, Lagrange multipliers, tangent cone, Dini differentiable functions, Hadamard differentiable functions, quasiconvex functions.

Mathematics Subject Classification. 90C29, 90C46.

\footnotetext{
Received December 5, 2003. Accepted March 25, 2004.

* This research for the second and third authors was partially supported by Ministerio de Ciencia y Tecnología (Spain), project BFM2003-02194.

1 Dipartimento di Ricerche Aziendali, Università degli Studi di Pavia, Via S. Felice, 5, 27100 Pavia, Italy; e-mail: ggiorgi@eco.unipv.it

2 Departamento de Economía e Historia Económica, Facultad de Economía y Empresa, Universidad de Salamanca, Campus Miguel de Unamuno, s/n, 37007 Salamanca, Spain; e-mail: bjimen1@encina.pntic.mec.es

3 Departamento de Matemática Aplicada, UNED, Calle Juan del Rosal, 12, Ciudad Universitaria, Apartado 60149, 28080 Madrid, Spain; e-mail: vnovo@ind.uned.es
} 


\section{INTRODUCTION}

Constraint qualifications have a significant role in optimization problems, since they allow us to guarantee the effective intervention of the objective function in the Fritz John type necessary conditions for a point to be an optimum. Since the first decade of the 50's, the study of these qualifications has been the aim of several researchers with different approaches, proposing various regularity conditions.

Maeda [10] studies multiobjective optimization problems with differentiable functions between finite-dimensional spaces and gives a Kuhn-Tucker type necessary condition for a Pareto optimum of a function over a feasible set defined by inequality constraints, assuring that the multipliers of the objective function are all positive under a regularity condition, called generalized Guignard constraint qualification. He also studies other qualifications, showing that this one is the weakest.

Preda and Chitescu [13] develop, at first, results similar to those obtained by Maeda, considering Dini-quasiconvex and directionally differentiable functions. But owing to the requirement on the objective functions to be Dini-quasiconvex and Dini-quasiconcave with convex and concave Dini derivatives, their necessary optimality conditions (Ths. 3.1 and 3.2) are very restrictive. On the other hand, the necessary condition expressed in Theorem 3.2, assuring the existence of positive multipliers for the objective functions, has a mistake that will be corrected in this paper.

Jiménez and Novo [7] extend the results obtained by Maeda for differentiable functions, by considering equality constraints, not considered by Maeda nor by Preda and Chitescu. They also introduced new qualifications that are sufficient conditions for what the afore mentioned papers called generalized Guignard constraint qualification.

In the present paper, the results obtained by Maeda, Preda and Chitescu and Jiménez and Novo are extended, by considering Dini or Hadamard differentiable functions and equality constraints. Furthermore, new qualifications are also introduced and the relationships between them are studied, thus obtaining a scheme which generalizes the ones of Bazaraa and Shetty [2], Figure 6.4, Maeda [10], Figure 1, Preda and Chitescu [13], Figure 1, and Jiménez and Novo [7], Figure 1.

This paper is structured as follows: Section 2 contains the definitions and notations we use and some previous results. In Section 3 several constraint qualifications are proposed and the relationships between them are studied. Finally, in Section 4, several necessary optimality conditions of the Kuhn-Tucker type are obtained, i.e. such that they assure the positivity of the multipliers under the weaker qualifications proposed. 


\section{NOTATIONS AND PRELIMINARIES}

Let $x$ and $y$ be two points of $\mathbb{R}^{n}$. Throughout this paper, we use the following notations.

$$
x \leq y \text { if } x_{i} \leq y_{i}, i=1, \ldots, n . x<y \text { if } x_{i}<y_{i}, i=1, \ldots, n .
$$

Let $S$ be a subset of $\mathbb{R}^{n}$. As usual, cl $S$, co $S$, cone $S$ and $\operatorname{lin} S$ will denote the closure, convex hull, generated cone and generated subspace by $S$, respectively. $B\left(x_{0}, \delta\right)$ is the open ball of center $x_{0}$ and radius $\delta>0$.

Given a function $f: \mathbb{R}^{n} \rightarrow \mathbb{R}^{p}$, the following multiobjective optimization problem is considered

$$
(\mathrm{MOP}) \operatorname{Min}\{f(x): x \in S\} \text {. }
$$

It is said that the point $x_{0} \in S$ is a local Pareto minimum, denoted $x_{0} \in$ $\operatorname{LMin}(f, S)$, if there exists a neighborhood of $x_{0}, B\left(x_{0}, \delta\right)$, such that

$$
S_{f} \cap S \cap B\left(x_{0}, \delta\right)=\emptyset,
$$

where $S_{f}=\left\{x \in \mathbb{R}^{n}: f(x) \leq f\left(x_{0}\right), f(x) \neq f\left(x_{0}\right)\right\}$.

The usual concepts of Pareto minimum, weak Pareto minimum and local weak Pareto minimum are also used. They will be denoted by $\operatorname{Min}(f, S), \operatorname{WMin}(f, S)$ and $\operatorname{LWMin}(f, S)$, respectively.

Because of the difficulties in verifying condition (1), different approximations at $x_{0}$ of the sets $S$ and $S_{f}$ are normally used, which have a simpler structure and are easier to obtain. The tangent cones are the approximations more usually used.

Definition 2.1. Let $S \subset \mathbb{R}^{n}, x_{0} \in \operatorname{cl} S$.

(a) The tangent cone to $S$ at the point $x_{0}$ is

$T\left(S, x_{0}\right)=\left\{v \in \mathbb{R}^{n}: \exists t_{k} \rightarrow 0^{+}, \exists x_{k} \in S\right.$ such that $\left.\left(x_{k}-x_{0}\right) / t_{k} \rightarrow v\right\}$.

(b) The cone of attainable directions is

$A\left(S, x_{0}\right)=\left\{v \in \mathbb{R}^{n}: \forall t_{k} \rightarrow 0^{+}, \exists x_{k} \in S\right.$ such that $\left.\left(x_{k}-x_{0}\right) / t_{k} \rightarrow v\right\}$.

(c) The cone of linear directions is

$Z\left(S, x_{0}\right)=\left\{v \in \mathbb{R}^{n}: \exists \delta>0\right.$ such that $\left.x_{0}+t v \in S \forall t \in(0, \delta]\right\}$.

For these cones, we have the following inclusions

$$
Z\left(S, x_{0}\right) \subset A\left(S, x_{0}\right) \subset T\left(S, x_{0}\right) .
$$

A complete and rigorous analysis of these cones in a greater detail can be found in Bazaraa and Shetty [2] and in Aubin and Frankowska [1].

Let $D \subset \mathbb{R}^{n}$. Then the polar cone to $D$ is $D^{*}=\left\{\xi \in \mathbb{R}^{n}:\langle\xi, d\rangle \leq 0 \forall d \in D\right\}$.

The normal cone to $S$ at $x_{0}$ is the polar to the tangent cone, i.e., $N\left(S, x_{0}\right)=$ $T\left(S, x_{0}\right)^{*}$.

Note that if the sets are defined through function constraints, their approximation is realized through the cones defined by the directional derivatives of the functions. 
Definition 2.2. Let $f: \mathbb{R}^{n} \rightarrow \mathbb{R}^{p}, x_{0}, v \in \mathbb{R}^{n}$.

(a) The Dini derivative (or directional derivative) of $f$ at $x_{0}$ in the direction $v$ is

$$
D f\left(x_{0}, v\right)=\lim _{t \rightarrow 0^{+}} \frac{f\left(x_{0}+t v\right)-f\left(x_{0}\right)}{t} .
$$

(b) The Hadamard derivative of $f$ at $x_{0}$ in the direction $v$ is

$$
d f\left(x_{0}, v\right)=\lim _{(t, u) \rightarrow\left(0^{+}, v\right)} \frac{f\left(x_{0}+t u\right)-f\left(x_{0}\right)}{t} .
$$

(c) $f$ is Dini differentiable (respectively Hadamard differentiable) at $x_{0}$ if its Dini derivative (resp. Hadamard derivative) exists for all the directions.

The following properties are well-known:

- if $f$ is Fréchet differentiable at $x_{0}$ with Fréchet differential $\nabla f\left(x_{0}\right)$, then $d f\left(x_{0}, v\right)=\nabla f\left(x_{0}\right) v$;

- if $d f\left(x_{0}, v\right)$ exists, then also $D f\left(x_{0}, v\right)$ exists and they are equal.

Definition 2.3. The Dini subdifferential of a Dini differentiable function $f: \mathbb{R}^{n} \rightarrow$ $\mathbb{R}$ at $x_{0}$ is

$$
\partial_{D} f\left(x_{0}\right)=\left\{\xi \in \mathbb{R}^{n}:\langle\xi, v\rangle \leq D f\left(x_{0}, v\right) \forall v \in \mathbb{R}^{n}\right\} .
$$

If $D f\left(x_{0}, v\right)$ is a convex function in $v$, then there exists the subdifferential (in the Convex Analysis sense) of this function at $v=0: \partial D f\left(x_{0}, \cdot\right)(0)$. This set is nonempty, compact and convex and $\partial_{D} f\left(x_{0}\right)=\partial D f\left(x_{0}, \cdot\right)(0)$.

In this paper, the following generalized convexity notions will be used.

Definition 2.4. Let $\Gamma \subset \mathbb{R}^{n}$ be a convex set, $f: \Gamma \rightarrow \mathbb{R}$, and $x_{0} \in \Gamma$.

(a) $f$ is quasiconvex at $x_{0}$ if $\forall x \in \Gamma, f(x) \leq f\left(x_{0}\right) \Rightarrow f\left(\lambda x+(1-\lambda) x_{0}\right) \leq$ $f\left(x_{0}\right) \forall \lambda \in(0,1)$

(b) $f$ is quasiconcave at $x_{0}$ if $-f$ is quasiconvex at $x_{0}$;

(c) $f$ is quasilinear at $x_{0}$ if $f$ is quasiconvex and quasiconcave at $x_{0}$;

(d) $f$ is pseudoconvex at $x_{0}$ if $\forall x \in \Gamma, f(x)<f\left(x_{0}\right) \Rightarrow D f\left(x_{0}, x-x_{0}\right)<0$;

(e) $f$ is pseudoconcave at $x_{0}$ if $-f$ is pseudoconvex at $x_{0} . f$ is pseudolinear at $x_{0}$ if $f$ is pseudoconvex and pseudoconcave at $x_{0}$;

(f) $f$ is linearlike at $x_{0}$ if $f(x)=f\left(x_{0}\right)+D f\left(x_{0}, x-x_{0}\right) \forall x \in \Gamma$;

(g) $f$ is Dini-quasiconvex at $x_{0}$ if $\forall x \in \Gamma, f(x) \leq f\left(x_{0}\right) \Rightarrow D f\left(x_{0}, x-x_{0}\right) \leq 0$;

(h) $f$ is Dini-quasilinear at $x_{0}$ if $f$ and $-f$ are Dini-quasiconvex at $x_{0}$;

(i) $f$ is quasiconvex on $\Gamma$ if $f$ is quasiconvex at each point of $\Gamma$. The other concepts here introduced can be defined on a set in a similar way.

In the next proposition we summarize some properties of the generalized convex functions previously introduced.

Proposition 2.1. Let $\Gamma \subset \mathbb{R}^{n}$ be a convex set, $f: \Gamma \rightarrow \mathbb{R}$, and $x_{0} \in \Gamma$.

(a) [3] Th. 3.5.2) $f$ is quasiconvex on $\Gamma$ if and only if the level sets $\Gamma_{\alpha}=\{x \in$ $\Gamma: f(x) \leq \alpha\}$ are convex for all $\alpha \in \mathbb{R}$. 
(b) Let $f$ be Dini differentiable at $x_{0}$. If $f$ is quasiconvex at $x_{0}$, then $f$ is Diniquasiconvex at $x_{0}$.

(c) ([5] Th. 3.5) if $f$ is pseudoconvex at $x_{0}$ and continuous on $\Gamma$, then $f$ is quasiconvex at $x_{0}$.

(d) ([5] Th. 3.2) if $f$ is continuous and Dini-quasiconvex on $\Gamma$, then $f$ is quasiconvex on $\Gamma$.

Remark 2.1. The following implications can easily be proved for a linear type function:

(i) if $f$ is linearlike at $x_{0}$, then $f$ is pseudolinear at $x_{0}$ and quasilinear at $x_{0}$;

(ii) if $f$ is quasilinear at $x_{0}$ and Dini differentiable at $x_{0}$, then $f$ is Diniquasilinear at $x_{0}$.

The second implication follows from Proposition 2.1(b). The converse of (i) does not hold. It can be proved, for instance, with the function $f: \mathbb{R} \rightarrow \mathbb{R}$ given by $f(x)=|x|+x^{2}$ and the point $x_{0}=0$.

Moreover, the reverse of Proposition 2.1(b) does not hold (i.e., if $f$ is Diniquasiconvex at $x_{0}$, then $f$ is quasiconvex at $x_{0}$ ), even if $f$ is differentiable at $x_{0}$. The function $f: \mathbb{R} \rightarrow \mathbb{R}$, given by $f(x)=x^{2} \sin (1 / x)$ if $x \neq 0$ and $f(0)=0$, is an evident counterexample for $x_{0}=0$.

Also, there is no in general implication relation between the concepts of pseudoconvexity at a point and quasiconvexity at a point. In fact, let $f: \mathbb{R}^{2} \rightarrow \mathbb{R}$ be the function given by

$$
f(x, y)=\left\{\begin{array}{l}
\|(x, y)\| \varphi\left(y / x^{2}\right) \text { if } x>0,1 \leq y / x^{2} \leq 3 \\
\|(x, y)\| \quad \text { otherwise }
\end{array}\right.
$$

where $\varphi: \mathbb{R} \rightarrow \mathbb{R}$ is defined by $\varphi(\alpha)=|\alpha-2|$ if $1 \leq \alpha \leq 3$ and $\varphi(\alpha)=1$ otherwise. Its Dini-derivative at $x_{0}=(0,0)$ is $D f\left(x_{0}, v\right)=\|v\| \forall v \in \mathbb{R}^{2}$. It follows that $f$ is pseudoconvex at $x_{0}$, since $\forall x \in \mathbb{R}^{2} f(x) \geq f\left(x_{0}\right)$, and it is not Dini-quasiconvex at $x_{0}$ over any neighborhood of $x_{0}$ (it is sufficient to consider the points $x_{n}=\left(\delta_{n}, \delta_{n}^{2}\right)$ with $\delta_{n} \rightarrow 0^{+}$, then $f\left(x_{n}\right)=f\left(x_{0}\right)$ and $\left.D f\left(x_{0}, x_{n}-x_{0}\right)>0\right)$. Hence, thanks to Proposition 2.1(b), $f$ is not quasiconvex at $x_{0}$. Furthermore, this function is pseudolinear at $x_{0}$ and, consequently, it is not true that a pseudolinear function at $x_{0}$ is quasilinear at $x_{0}$ (or Dini-quasilinear at $x_{0}$ ).

The function $f(x)=x^{3}$ is quasiconvex at $x_{0}=0$ but it is not pseudoconvex.

Finally, none of the linear types guarantees by itself the continuity of the derivative. Example 3.1 in [4] Chapter 1 shows this fact.

We say that the convex sets $B_{j}, j \in J=\{1, \ldots, m\}$, of $\mathbb{R}^{n}$ are positively linearly independent (p.l.i.) if

$$
0 \in \sum_{j \in J} \lambda_{j} B_{j}, \lambda \geq 0 \Rightarrow \lambda=0,
$$

i.e., if $0 \notin \operatorname{co}\left(\cup_{j \in J} B_{j}\right)$. 
In Section 4 necessary conditions for a local Pareto minimum with positive multipliers for the objective functions will be obtained. It is however necessary to establish first a Tucker type alternative theorem [11] Theorem 3, Chapter 2.4. We choose the version obtained by Ishizuka [6] Proposition 2.2 in a simplified form, and we give it in a suitable form for our purposes.

Proposition 2.2 (generalized Tucker alternative theorem). Let $f_{1}, \ldots, f_{p}, g_{1}, \ldots$, $g_{m}$ be sublinear functions from $\mathbb{R}^{n}$ to $\mathbb{R}$ and $h_{1}, \ldots, h_{r}$ linear functions from $\mathbb{R}^{n}$ to $\mathbb{R}$ given by $h_{k}(v)=\left\langle c_{k}, v\right\rangle, k \in K=\{1, \ldots, r\}$. Suppose that for each $i \in\{1, \ldots, p\}$ the cone

$$
D_{i}=\operatorname{coneco}\left(\cup_{j \neq i} \partial f_{j}(0)\right)+\operatorname{cone} \operatorname{co}\left(\cup_{j=1}^{m} \partial g_{j}(0)\right)+\operatorname{lin}\left\{c_{k}: k \in K\right\}
$$

is closed. Then, the following statements are equivalent:

(a) The system

$$
f(v) \leq 0, f(v) \neq 0, g(v) \leq 0, h(v)=0
$$

has no solution $v \in \mathbb{R}^{n}$.

(b) There exist $(\lambda, \mu, \nu) \in \mathbb{R}^{p} \times \mathbb{R}^{m} \times \mathbb{R}^{r}$ such that $\lambda>0$, $\mu \geq 0$ and

$$
0 \in \sum_{i=1}^{p} \lambda_{i} \partial f_{i}(0)+\sum_{j=1}^{m} \mu_{j} \partial g_{j}(0)+\sum_{k=1}^{r} \nu_{k} c_{k} .
$$

Proof. To transform this proposition into the one of Ishizuka, it is enough to consider $A_{i}=\partial f_{i}(0), i=1, \ldots, p, B_{j}=\partial g_{j}(0), j=1, \ldots, m, B_{m+k}=\operatorname{co}\left\{-c_{k}, c_{k}\right\}=$ $\left[-c_{k}, c_{k}\right], k=1, \ldots, r$, which implies $f_{i}(v)=\operatorname{Max}_{a \in A_{i}}\langle a, v\rangle, i=1, \ldots, p, g_{j}(v)=$ $\operatorname{Max}_{b \in B_{j}}\langle b, v\rangle, j=1, \ldots, m$. Let

$$
g_{m+k}(v)=\operatorname{Max}_{c \in B_{m+k}}\langle c, v\rangle=\operatorname{Max}\left\{-\left\langle c_{k}, v\right\rangle,\left\langle c_{k}, v\right\rangle\right\}=\left|h_{k}(v)\right|, k=1, \ldots, r .
$$

We have $\partial g_{m+k}(0)=B_{m+k}$ and the equation $h_{k}(v)=0$ is equivalent to $g_{m+k}(v) \leq$ 0 . By means of this notation, as the cones $D_{i}$ are closed, according to Ishizuka's Proposition 2.2 [6], (a) is equivalent to

(c) There exist $(\lambda, \mu, \alpha) \in \mathbb{R}^{p} \times \mathbb{R}^{m} \times \mathbb{R}^{r}$ such that $\lambda>0,(\mu, \alpha) \geq 0$ and $0 \in \sum_{i=1}^{p} \lambda_{i} \partial f_{i}(0)+\sum_{j=1}^{m} \mu_{j} \partial g_{j}(0)+\sum_{k=1}^{r} \alpha_{k}\left[-c_{k}, c_{k}\right]$

Taking into account that $c \in \alpha_{k}\left[-c_{k}, c_{k}\right]$ for some $\alpha_{k} \geq 0$ if and only if there exists $\nu_{k} \in \mathbb{R}$ such that $c=\nu_{k} c_{k}$, proposition (c) is equivalent to (b).

In order to decide if the cones $D_{i}$ are closed, we have the following criterium.

Remark 2.2. Note that if $0 \notin \operatorname{co}\left(\cup_{j \neq i} A_{j} \cup \cup_{j=1}^{m} B_{j}\right)+\operatorname{lin}\left\{c_{k}: k \in K\right\}$, then $D_{i}$ is closed. This follows from Proposition 3.6 in [8].

Note that if $0 \notin C=\operatorname{co}\left(\cup_{i=1}^{p} A_{i} \cup \cup_{j=1}^{m} B_{j}\right)+\operatorname{lin}\left\{c_{k}: k \in K\right\}$, then the $p$ cones $D_{i}$ are closed. But this condition is incompatible with Proposition 2.2(b) and, consequently, with Proposition (a). As a matter of fact, if $0 \notin C$ and $u=$ $\operatorname{Proy}_{C}(0)$, then the vector $v=-u$ is a solution of the system in (a). 
Now we consider a set $S$ defined by equality and inequality constraints and a point of $S$ at which we need to obtain the tangent cone. This is done in Proposition 2.6.

From now on, we shall assume that the feasible set of problem (MOP) is defined by

$$
S=\left\{x \in \mathbb{R}^{n}: g(x) \leq 0, h(x)=0\right\},
$$

where $g: \mathbb{R}^{n} \rightarrow \mathbb{R}^{m}$ and $h: \mathbb{R}^{n} \rightarrow \mathbb{R}^{r}$, whose component functions are, respectively, $g_{j}, j \in J=\{1, \ldots, m\}, h_{k}, k \in K=\{1, \ldots, r\}$. We shall adopt the following notation. Given $x_{0} \in S$, the active index set at $x_{0}$ is $J_{0}=\left\{j \in J: g_{j}\left(x_{0}\right)=\right.$ $0\}$. The sets defined by the constraints $g$ and $h$ are denoted, respectively, by $G=\left\{x \in \mathbb{R}^{n}: g(x) \leq 0\right\}, H=\left\{x \in \mathbb{R}^{n}: h(x)=0\right\}$, so $S=G \cap H$.

We suppose that all functions considered are continuous at $x_{0}$ and that the active constraints are Dini differentiable at $x_{0}$. The cones that we shall use in order to approximate $S$ at $x_{0}$ are (linearized cones):

$$
\begin{aligned}
C_{0}(S) & =\left\{v \in \mathbb{R}^{n}: D g_{j}\left(x_{0}, v\right)<0 \forall j \in J_{0}, D h_{k}\left(x_{0}, v\right)=0 \forall k \in K\right\} \\
C(S) & =\left\{v \in \mathbb{R}^{n}: D g_{j}\left(x_{0}, v\right) \leq 0 \forall j \in J_{0}, D h_{k}\left(x_{0}, v\right)=0 \forall k \in K\right\} .
\end{aligned}
$$

$C_{0}(G)$ and $C(G)$ are defined in an analogous way and we denote $K(H)=\operatorname{Ker} D h\left(x_{0}, \cdot\right)$. Consequently, $C_{0}(S)=C_{0}(G) \cap K(H)$ and $C(S)=$ $C(G) \cap K(H)$.

Our aim is to obtain the inclusions

$$
C_{0}(S) \subset T\left(S, x_{0}\right) \subset C(S)
$$

This is done in the following propositions.

Proposition $2.3\left([12]\right.$, (Prop. 3.1)). Let $D g_{j}\left(x_{0}, \cdot\right), j \in J_{0}$ be convex, Dh $\left(x_{0}, \cdot\right)$ linear and $C_{0}(S) \neq \emptyset$. Then $\operatorname{cl} C_{0}(S)=C(S)$.

Proposition 2.4. Suppose that for each $j \in J_{0}$, either $g_{j}$ is Hadamard differentiable at $x_{0}$ or $g_{j}$ is Dini-quasiconvex at $x_{0}$ and $D g_{j}\left(x_{0}, \cdot\right)$ is continuous on $\mathbb{R}^{n}$, and for each $k \in K$, either $h_{k}$ is Hadamard differentiable at $x_{0}$ or Dini-quasilinear at $x_{0}$ with $D h_{k}\left(x_{0}, \cdot\right)$ continuous. Then

$$
T\left(S, x_{0}\right) \subset C(S)
$$

The proof of the previous proposition is similar to that of Lemma 3.2 in [9].

Proposition 2.5. If there is no equality constraints, $S=G$, and the functions $g_{j}, j \in J_{0}$, are Dini differentiable at $x_{0}$, then

$$
C_{0}(G) \subset Z\left(G, x_{0}\right) \subset\left\{\begin{array}{l}
A\left(G, x_{0}\right) \subset T\left(G, x_{0}\right) \\
C(G) .
\end{array}\right.
$$


Proposition 2.6 [9] (Cor. 3.5). Let us suppose the following:

(a) $h$ is continuous on a neighborhood of $x_{0}$, Fréchet differentiable at $x_{0}$ and $\left\{\nabla h_{k}\left(x_{0}\right): k \in K\right\}$ is linearly independent;

(b) for each $j \in J_{0}, g_{j}$ is either Dini-quasiconvex and continuous on a neighborhood of $x_{0}$ or Hadamard differentiable at $x_{0}$, in both cases with convex derivative at $x_{0}$; (c) $C_{0}(S) \neq \emptyset$.

Then

$$
\operatorname{cl} C_{0}(S)=A\left(S, x_{0}\right)=T\left(S, x_{0}\right)=C(S) .
$$

Note that, by [8], Theorem 3.9, we have that $\left\{\nabla h_{k}\left(x_{0}\right): k \in K\right\}$ is linearly independent and $C_{0}(S) \neq \emptyset$ if and only if the following implication is true:

$$
0 \in \sum_{j \in J_{0}} \mu_{j} \partial_{D} g_{j}\left(x_{0}\right)+\sum_{k=1}^{r} \nu_{k} \nabla h_{k}\left(x_{0}\right), \mu \geq 0 \Rightarrow \mu=0, \nu=0,
$$

which is constraint qualification (CQ2) in [9]. By Proposition 2.1(d), if $g_{j}$ is Diniquasiconvex and continuous on a neighborhood of $x_{0}$, then $g_{j}$ is quasiconvex on such a neighborhood.

\section{Constraint qualifications in multiobjective OPTIMIZATION}

Let us consider the multiobjective optimization problem

$$
\text { (MOP) } \operatorname{Min}\{f(x): x \in S\} \text {, }
$$

where the feasible set $S$ is given by (3) and $f: \mathbb{R}^{n} \rightarrow \mathbb{R}^{p}$ has component functions $f_{i}, i \in I=\{1, \ldots, p\}$.

By keeping the notation of Section 2, given $x_{0} \in S$, the following sets are considered: $F=\left\{x: f(x) \leq f\left(x_{0}\right)\right\}, S^{0}=F \cap S$ and for each $i \in I, F^{i}=$ $\left\{x: f_{j}(x) \leq f_{j}\left(x_{0}\right) \forall j \in I \backslash\{i\}\right\}$ and $S^{i}=F^{i} \cap S$. Obviously $F=\cap_{i=1}^{p} F^{i}$ and $S^{0}=\cap_{i=1}^{p} S^{i}$. Since the sets given above are defined by function constraints, the corresponding linearized cones can be defined. Let us remark that for the set $F$ all functions $f_{i}, i \in I$, are active at $x_{0}$ and for the set $F^{i}$ the same is true for the functions $f_{j}, j \in I \backslash\{i\}$. We have $C_{0}\left(S^{i}\right)=C_{0}\left(F^{i}\right) \cap C_{0}(G) \cap K(H)$, $C\left(S^{i}\right)=C\left(F^{i}\right) \cap C(G) \cap K(H)$ and similar expressions for $C_{0}\left(S^{0}\right)$ and $C\left(S^{0}\right)$.

It is a known result that $x_{0}$ is a local Pareto minimum to problem (MOP) if and only if for each $i=1, \ldots, p, x_{0}$ is a local minimum of the scalar problem

$$
\left(\mathrm{P}_{i}\right) \operatorname{Min}\left\{f_{i}(x): x \in S^{i}\right\} .
$$

We consider now different qualifications for problem (MOP) in the approaches of Maeda [10], Preda and Chitescu [13] and Jiménez and Novo [7]. The implications between the various qualifications are also analyzed. 
We suppose that all functions are Dini differentiable at $x_{0}$, unless we specify another thing.

Let us consider the following hypotheses:

(H0) $h$ is continuous on a neighborhood of $x_{0}$. Fréchet differentiable at $x_{0}$ and $\left\{\nabla h_{k}\left(x_{0}\right): k \in K\right\}$ is linearly independent.

(H1) Each function of the set $\left\{f_{i}, g_{j}: i \in I, j \in J_{0}\right\}$ is either Dini-quasiconvex at $x_{0}$ with continuous derivative on $\mathbb{R}^{n}$ or Hadamard differentiable at $x_{0}$.

(H2) Each function of the set $\left\{f_{i}, g_{j}: i \in I, j \in J_{0}\right\}$ is either Dini-quasiconvex and continuous on a neighborhood of $x_{0}$ or Hadamard differentiable at $x_{0}$.

(H3) For each $i=1, \ldots, p, T\left(S^{i}, x_{0}\right) \subset C\left(S^{i}\right)$ holds true.

(H4) Each function in the set $\left\{D f_{i}\left(x_{0}, \cdot\right), D g_{j}\left(x_{0}, \cdot\right): i \in I, j \in J_{0}\right\}$ is convex.

Definition 3.1. The next constraint qualifications are considered:

1. Generalized Guignard (GGCQ): $C\left(S^{0}\right)=\cap_{i=1}^{p} \operatorname{clco} T\left(S^{i}, x_{0}\right)$.

2. Abadie $(A C Q): C\left(S^{0}\right)=T\left(S^{0}, x_{0}\right)$ and $(\mathrm{H} 3)$.

3. Generalized Abadie (GACQ): $C\left(S^{0}\right)=\cap_{i=1}^{p} T\left(S^{i}, x_{0}\right)$ and (H3).

4. Global Cottle (GCCQ): $C_{0}(F) \cap C_{0}(S) \neq \emptyset,(\mathrm{H} 0)$ and $(\mathrm{H} 4)$.

5. Cottle $(C C Q)$ : for each $i=1 \ldots p, C_{0}\left(S^{i}\right) \neq \emptyset(\mathrm{H} 0)$ and $(\mathrm{H} 4)$.

6. Slater type.

a) Slater $(S C Q): f_{i}, i \in I, g_{j}, j \in J_{0}$, are pseudoconvex at $x_{0} ; h_{k}, k \in K$, are Dini-quasilinear at $x_{0},(\mathrm{H} 0),(\mathrm{H} 4)$ and for each $i=1, \ldots, p$ there exists $x_{i} \in \mathbb{R}^{n}$ such that

$f_{j}\left(x_{i}\right)<f_{j}\left(x_{0}\right) \forall j \neq i, g_{j}\left(x_{i}\right)<0 \forall j \in J_{0}$, and $h_{k}\left(x_{i}\right)=0 \forall k \in K$.

b) Differentiable Slater $(D S C Q): f_{i}, i \in I, g_{j}, j \in J_{0}$, are pseudoconvex at $x_{0},(\mathrm{H} 0),(\mathrm{H} 4)$ and for each $i=1, \ldots, p$ there exists $x_{i} \in \mathbb{R}^{n}$ such that

$$
f_{j}\left(x_{i}\right)<f_{j}\left(x_{0}\right) \forall j \neq i, g_{j}\left(x_{i}\right)<0 \forall j \in J_{0} \text {, and } x_{i}-x_{0} \in K(H) .
$$

7. Linearlike (LLCQ): $f_{i}, g_{j}, h_{k}, i \in I, j \in J_{0}, k \in K$, are all linearlike at $x_{0}$ with continuous derivative.

8. Linearlike objectives ( $L L O): f_{i}, i \in I$, are linearlike at $x_{0}$ with convex derivative, each $g_{j}, j \in J_{0}$, has convex derivative and is either Hadamard differentiable or Dini-quasiconvex at $x_{0}, h_{k}, k \in K$, are affine and $C(F) \cap C_{0}(G) \cap K(H) \neq \emptyset$. 9. Mangasarian-Fromovitz. Each qualification in this group must verify (H0) and (H4):

a) With positively linearly independent objectives (PIOMF): $C(F) \cap C_{0}(S) \neq$ $\emptyset$ and $C_{0}(F) \cap K(H) \neq \emptyset$.

b) With quasiindependent objectives $(Q I O M F): C(F) \cap C_{0}(S) \neq \emptyset$ and for each $i=1, \ldots, p$ we have that $C_{0}\left(F^{i}\right) \cap K(H) \neq \emptyset$.

c) With positively linearly independent constraints (PICMF): $C_{0}(F) \cap$ $C(S) \neq \emptyset$ and $C_{0}(G) \cap K(H) \neq \emptyset$.

d) With $C(F) \cap C_{0}(G) \cap K(H) \neq \emptyset$ and for each $i=1, \ldots, p, C(F) \cap$ $C_{0}(G) \cap K(H) \not \subset \operatorname{Ker} D f_{i}\left(x_{0}, \cdot\right)$. We speak in this case of Preda-Chitescu Mangasarian-Fromovitz qualification (PCMF). 
10. Zangwill $(Z C Q): \operatorname{cl} Z\left(S^{0}, x_{0}\right)=C\left(S^{0}\right)$ and $(\mathrm{H} 3)$.

11. Kuhn-Tucker $(K T C Q): A\left(S^{0}, x_{0}\right)=C\left(S^{0}\right)$ and $(\mathrm{H} 3)$.

12. Reverse $(R C Q): f_{i}, g_{j}, i \in I, j \in J_{0}$, are pseudoconcave at $x_{0},(\mathrm{H} 1)$ and $h_{k}, k \in K$, are linearlike at $x_{0}$ with continuous derivative.

\section{Lemma 3.1.}

(i) If $h$ is linearlike at $x_{0}$ with continuous Dini derivative, then $h$ is Hadamard differentiable at $x_{0}$.

(ii) If $h$ is pseudolinear and Dini-quasilinear at $x_{0}$, then

(a) $Z\left(H, x_{0}\right)=T\left(H, x_{0}\right)=K(H)$;

(b) $H=x_{0}+K(H)$.

(iii) If $g_{j}, j \in J_{0}$, are pseudoconcave at $x_{0}$, then $Z\left(G, x_{0}\right)=C(G)$.

Proof.

(i) It is an elementary exercise.

(ii) (a) $Z\left(H, x_{0}\right) \subset T\left(H, x_{0}\right)$ is true for all sets $H$ and $T\left(H, x_{0}\right) \subset K(H)$ by Proposition 2.4. We now prove that $K(H) \subset Z\left(H, x_{0}\right)$. Let $v \in K(H)$, then $D h\left(x_{0}, v\right)=0$ and therefore $D h\left(x_{0},\left(x_{0}+t v\right)-x_{0}\right) \geq 0 \forall t>0$. Since $h$ is pseudoconvex, $h\left(x_{0}+t v\right) \geq h\left(x_{0}\right)=0 \forall t>0$, and analogously, due to the pseudoconcavity, $h\left(x_{0}+t v\right) \leq h\left(x_{0}\right)=0$. Consequently $h\left(x_{0}+t v\right)=0$, i.e., $x_{0}+t v \in H \forall t>0$, which implies $v \in Z\left(H, x_{0}\right)$.

(b) We have just proved that $x_{0}+t v \in H \forall t>0$. Taking $t=1$, we have $v \in H-x_{0}$ and thus $K(H) \subset H-x_{0}$. Now we prove the reverse inclusion. Let $x \in H$, hence $h(x)-h\left(x_{0}\right) \leq 0$. Since $h$ is Dini-quasiconvex at $x_{0}$, it follows $D h\left(x_{0}, x-x_{0}\right) \leq 0$. Likewise with $-h$, we get $-D h\left(x_{0}, x-x_{0}\right) \leq 0$. Consequently, $\operatorname{Dh}\left(x_{0}, x-x_{0}\right)=0$, which means, $x-x_{0} \in K(H)$.

(iii) From Proposition 2.5, we only have to prove that $C(G) \subset Z\left(G, x_{0}\right)$. Let $v \in C(G)$, then $D g_{j}\left(x_{0}, v\right) \leq 0 \forall j \in J_{0}$. By pseudoconcavity, $g_{j}\left(x_{0}+t v\right) \leq$ $g_{j}\left(x_{0}\right)=0 \forall t>0$. If $j \in J \backslash J_{0}$, by the continuity of $g_{j}$ we have $g_{j}\left(x_{0}+t v\right)<0$ for all $t$ small enough. Therefore, $x_{0}+t v \in G$, and consequently $v \in Z\left(G, x_{0}\right)$, thus completing the proof.

We remark that just by using the definition, we get that if $h$ is linearlike at $x_{0}$ with linear Dini derivative, then $h$ is affine. If $h$ is linearlike at $x_{0}$ with continuous Dini derivative, then part (ii) of Lemma 3.1 holds true (according to Rem. 2.1, $h$ is pseudolinear and Dini-quasilinear at $x_{0}$ ).

In Theorem 3.1 below, the relationship between the different constraint qualifications are established. In order to prove the theorem we need a previous lemma. The inclusion relationships in the lemma are obvious and the proof of the second part is similar to that of Proposition 2.3.

Lemma 3.2. If $D h\left(x_{0}, \cdot\right)$ is linear and $D f_{i}\left(x_{0}, \cdot\right), D g_{j}\left(x_{0}, \cdot\right), i \in I, j \in J_{0}$ are convex, then

$$
\begin{aligned}
C_{0}\left(S^{0}\right)=C_{0}(F) \cap C_{0}(G) \cap K(H) \subset\left\{\begin{array}{l}
C(F) \cap C_{0}(G) \cap K(H) \\
C_{0}(F) \cap C(G) \cap K(H)
\end{array}\right\} \\
\subset C(F) \cap C(G) \cap K(H)=C\left(S^{0}\right),
\end{aligned}
$$




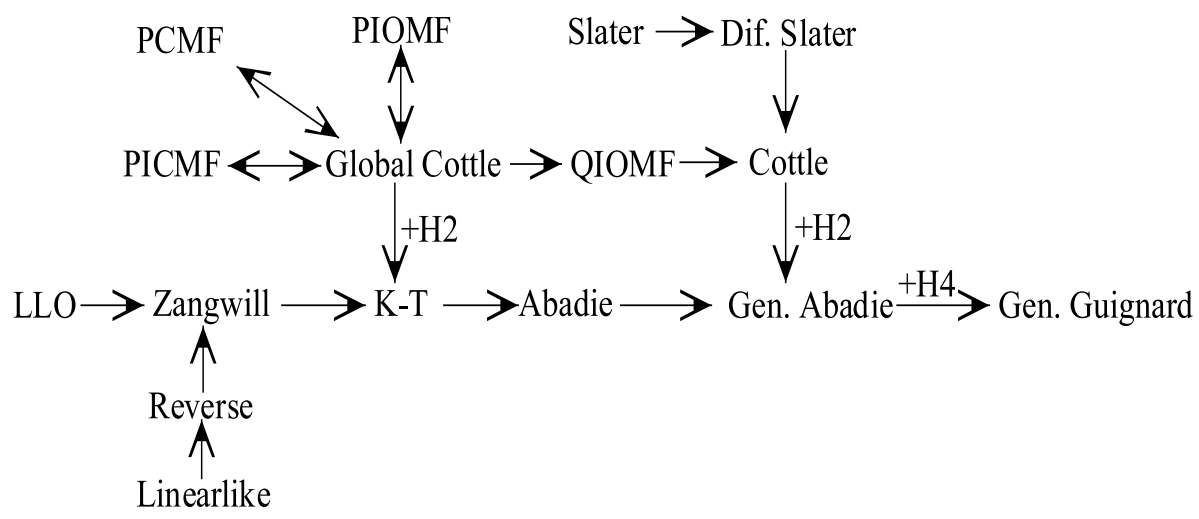

FiguRE 1. Relationship between the constraint qualifications.

and if some of the sets $C_{0}\left(S^{0}\right), C(F) \cap C_{0}(G) \cap K(H)$ and $C_{0}(F) \cap C(G) \cap K(H)$ is nonempty then its closure is $C\left(S^{0}\right)$.

Theorem 3.1. The following implications are verified:

1. Linearlike $\Rightarrow$ Reverse $\Rightarrow$ Zangwill.

2. Linearlike objectives $\Rightarrow$ Zangwill.

3. Slater $\Rightarrow$ Differentiable Slater $\Rightarrow$ Cottle.

4. $P I C M F \Leftrightarrow$ Global Cottle $\Leftrightarrow P I O M F \Leftrightarrow P C M F$.

5. Global Cottle $\Rightarrow$ QIOMF $\Rightarrow$ Cottle.

6. Cottle and (H2) $\Rightarrow$ Generalized Abadie.

7. Global Cottle and (H2) $\Rightarrow$ Kuhn-Tucker.

8. a) Zangwill $\Rightarrow$ Kuhn-Tucker $\Rightarrow$ Abadie $\Rightarrow$ Generalized Abadie.

b) Generalized Abadie and (H4) $\Rightarrow$ Generalized Guignard.

The results above are summarized in Figure 1, which generalizes the similar figures in [2], Figure 6.4, [10], Figure 1, [13], Figure 1 and [7], Figure 1.

Proof.

1. a) Linearlike $\Rightarrow$ Reverse. It suffices to observe that if a function is linearlike, then it is pseudoconcave and, since it has continuous derivative, by Lemma 3.1(i), it follows that it is Hadamard differentiable, which implies (H1).

b) Reverse $\Rightarrow$ Zangwill. Lemma 3.1(iii) shows that $Z\left(F \cap G, x_{0}\right)=C(F \cap G)$, and by Lemma $3.1(\mathrm{ii}), Z\left(H, x_{0}\right)=K(H)$. Thus

$$
\begin{aligned}
Z\left(S^{0}\right) & =Z\left(F \cap G \cap H, x_{0}\right)=Z\left(F \cap G, x_{0}\right) \cap Z\left(H, x_{0}\right) \\
& =C(F \cap G) \cap K(H)=C\left(S^{0}\right) .
\end{aligned}
$$

Since (H1) is true, (H3) follows from Proposition 2.4.

2. Linearlike objectives $\Rightarrow$ Zangwill. From Proposition 2.5 it follows $C_{0}(G) \subset$ $Z\left(G, x_{0}\right)$. Since $f$ is linearlike at $x_{0}$, by means of Lemma 3.1(iii), we get $Z\left(F, x_{0}\right)=$ 
$C(F)$. As $h$ is affine, $Z\left(H, x_{0}\right)=K(H)$. Hence we have

$C(F) \cap C_{0}(G) \cap K(H) \subset Z\left(F, x_{0}\right) \cap Z\left(G, x_{0}\right) \cap Z\left(H, x_{0}\right)=Z\left(S^{0}, x_{0}\right) \subset T\left(S^{0}, x_{0}\right)$.

As $f$ is linearlike with continuous derivative, from Lemma 3.1(i), $f$ is Hadamard differentiable. Since $g_{j}, j \in J_{0}$, is Hadamard differentiable or Dini-quasiconvex, by Proposition 2.4, $T\left(S^{i}, x_{0}\right) \subset C\left(S^{i}\right) \forall i \in I$ and $T\left(S^{0}, x_{0}\right) \subset C\left(S^{0}\right)$. Taking this last inclusion and (7) into account and using Lemma 3.2, we can conclude that $\operatorname{cl} Z\left(S^{0}, x_{0}\right)=C\left(S^{0}\right)$.

3. a) Slater $\Rightarrow$ Differentiable Slater. For each problem $\left(\mathrm{P}_{i}\right)$ there exists $x_{i}$ verifying (5). In particular, $h_{k}\left(x_{i}\right)=h_{k}\left(x_{0}\right)$, and by the Dini-quasiconvexity of $h_{k}$ and $-h_{k}$, $\nabla h_{k}\left(x_{0}\right)\left(x_{i}-x_{0}\right)=0, \forall k \in K$, which means that $x_{i}-x_{0} \in K(H)$.

b) Differentiable Slater $\Rightarrow$ Cottle. For each $i=1, \ldots, p$, there exists $x_{i}$ verifying (6). Because of the pseudoconvexity of $f_{j}$ and $g_{j}$, we have

$$
D f_{j}\left(x_{0}, x_{i}-x_{0}\right)<0 \forall j \neq i, D g_{j}\left(x_{0}, x_{i}-x_{0}\right)<0 \forall j \in J_{0} .
$$

By hypothesis, $x_{i}-x_{0} \in K(H)$, and consequently $x_{i}-x_{0} \in C_{0}\left(S^{i}\right)$.

4. a) $\mathrm{PICMF} \Rightarrow$ Global Cottle. By assumption we have $C_{0}(G) \cap K(H) \neq \emptyset$, and hence from Proposition 2.3

$$
\operatorname{cl}\left[C_{0}(G) \cap K(H)\right]=C(G) \cap K(H) .
$$

Let $v \in C_{0}(F) \cap[C(G) \cap K(H)]$ (this set is nonempty by assumption). Since $C_{0}(F)$ is open, there exists a neighborhood $B(v)$ of $v$, such that $B(v) \subset C_{0}(F)$, and from (8), $B(v) \cap\left[C_{0}(G) \cap K(H)\right] \neq \emptyset$. So, we can state that $C_{0}(F) \cap C_{0}(G) \cap K(H) \neq \emptyset$ and hence we have the global Cottle qualification.

b) Global Cottle $\Rightarrow$ PICMF. By hypothesis, $C_{0}(F) \cap C_{0}(G) \cap K(H) \neq \emptyset$, and therefore $C_{0}(F) \cap C(S) \neq \emptyset$ and $C_{0}(G) \cap K(H) \neq \emptyset$, that is, we have PICMF.

c) Global Cottle $\Leftrightarrow$ PIOMF. It is enough to note that $f$ and $g$ have a symmetric role in PIOMF and PICMF. So, if PICMF $\Leftrightarrow$ Global Cottle, then also PIOMF $\Leftrightarrow$ Global Cottle.

d) Global Cottle $\Leftrightarrow$ PCMF.

$[\Rightarrow]$ It is immediate, because there exists $v \in C_{0}(F) \cap C_{0}(G) \cap K(H)$. Then, $D f_{i}\left(x_{0}, v\right)<0 \forall i \in I$.

$[\Leftarrow]$ By hypothesis, $\forall i \in I \exists v_{i} \in C(F) \cap C_{0}(G) \cap K(H)$ such that $D f_{i}\left(x_{0}, v_{i}\right)<0$. Let be $v=\sum_{i=1}^{p} \lambda_{i} v_{i}$ with $\lambda_{i}=1 / p$. We shall see that $v \in C_{0}(F) \cap C_{0}(G) \cap K(H)$, which implies global Cottle.

By the convexity of $D f_{j}\left(x_{0}, \cdot\right)$ we have $D f_{j}\left(x_{0}, v\right) \leq \sum_{i=1}^{p} \lambda_{i} D f_{j}\left(x_{0}, v_{i}\right)<0$, since $D f_{j}\left(x_{0}, v_{i}\right) \leq 0 \forall j \neq i$ and $D f_{i}\left(x_{0}, v_{i}\right)<0$. Analogously, $D g_{j}\left(x_{0}, v\right)<0 \forall j \in J_{0}$ and by the linearity of $\nabla h_{k}\left(x_{0}\right), \nabla h_{k}\left(x_{0}\right) v=0$.

5. a) Global Cottle $\Rightarrow$ QIOMF. As global Cottle is equivalent to PIOMF, it is enough to get that PIOMF implies QIOMF. But this is obvious, because if $C_{0}(F) \cap K(H) \neq \emptyset$, then for each $i=1, \ldots, p C_{0}\left(F^{i}\right) \cap K(H) \neq \emptyset$. 
b) QIOMF $\Rightarrow$ Cottle. Assume that there exists $i \in I$ such that $C_{0}\left(S^{i}\right)=\emptyset$. This means that there is no solution $v \in \mathbb{R}^{n}$ of the system

$$
\left\{\begin{array}{l}
D f_{j}\left(x_{0}, v\right)<0 \forall j \neq i \\
D g_{j}\left(x_{0}, v\right)<0 \forall j \in J_{0} \\
\nabla h_{k}\left(x_{0}\right) v=0 \forall k \in K .
\end{array}\right.
$$

Using Theorem 3.5 in $[8]$ we obtain that there exists $(\lambda, \mu, \nu) \in \mathbb{R}^{p-1} \times \mathbb{R}^{J_{0}} \times \mathbb{R}^{r}$ such that $(\lambda, \mu) \geq 0,(\lambda, \mu) \neq 0$ and

$$
\sum_{j \neq i} \lambda_{j} D f_{j}\left(x_{0}, v\right)+\sum_{j \in J_{0}} \mu_{j} D g_{j}\left(x_{0}, v\right)+\sum_{k=1}^{r} \nu_{k} \nabla h_{k}\left(x_{0}\right) v \geq 0 \forall v \in \mathbb{R}^{n} .
$$

By hypothesis, there exists $u \in C(F) \cap C_{0}(G) \cap K(H)$. If for some $j \in J_{0}$, $\mu_{j}>0$, then $\sum_{j \neq i} \lambda_{j} D f_{j}\left(x_{0}, u\right)+\sum_{j \in J_{0}} \mu_{j} D g_{j}\left(x_{0}, u\right)<0$, in contrast with the result obtained in (9) with $v=u$. Thus $\mu=0$ and in (9) we have therefore that $\sum_{j \neq i} \lambda_{j} D f_{j}\left(x_{0}, v\right)+\sum_{k=1}^{r} \nu_{k} \nabla h_{k}\left(x_{0}\right) v \geq 0 \forall v \in \mathbb{R}^{n}$. By hypothesis, there exists $w \in C_{0}\left(F^{i}\right) \cap K(H)$ and an analogous argument shows that $\lambda=0$, which is a contradiction.

6. Cottle and $(\mathrm{H} 2) \Rightarrow$ Generalized Abadie. It is enough to apply Proposition 2.6 to each set $S^{i}$.

7. Global Cottle and (H2) $\Rightarrow$ Kuhn-Tucker. To prove this result, it is sufficient to take the implication Global Cottle $\Rightarrow$ Cottle into account and to apply Proposition 2.6 to each $S^{i}$ and to $S^{0}$.

8. a) Zangwill $\Rightarrow$ Kuhn-Tucker $\Rightarrow$ Abadie $\Rightarrow$ Generalized Abadie. Since $S^{0} \subset$ $S^{i} \forall i \in I$, from the isotonicity of the tangent cone and from (H3) it follows that

$$
T\left(S^{0}, x_{0}\right) \subset \cap_{i=1}^{p} T\left(S^{i}, x_{0}\right) \subset \cap_{i=1}^{p} C\left(S^{i}\right)=C\left(S^{0}\right) .
$$

Now, from equation (2) applied to $S^{0}$, the three implications follow.

b) Generalized Abadie and (H4) $\Rightarrow$ Generalized Guignard. Obviously, $T\left(S^{i}, x_{0}\right) \subset$ $\operatorname{cl} \operatorname{co} T\left(S^{i}, x_{0}\right) \subset C\left(S^{i}\right)$ (the last inclusion is due to the convexity of the derivatives and to (H3)). Therefore we get

$$
\cap_{i=1}^{p} T\left(S^{i}, x_{0}\right) \subset \cap_{i=1}^{p} \operatorname{cl} \operatorname{co} T\left(S^{i}, x_{0}\right) \subset \cap_{i=1}^{p} C\left(S^{i}\right)=C\left(S^{0}\right)
$$

and the implication is then evident.

\section{Remark 3.1.}

(1) It is known that if $f$ is Hadamard differentiable at $x_{0}$ and $x_{0}$ is a local Pareto minimum of $f$ over $S$, then

$$
C_{0}(F) \cap T\left(S, x_{0}\right)=\emptyset \text {. }
$$


(2) If for some $i \in I, f_{i}$ is Hadamard differentiable at $x_{0},(\mathrm{H} 0),(\mathrm{H} 2)$ and (H4) hold, and $x_{0} \in \operatorname{LMin}(f, S)$, then $C_{0}\left(S^{0}\right)=\emptyset$ (and consequently, Global Cottle qualification is not satisfied at $\left.x_{0}\right)$.

Indeed one has that $x_{0}$ is a local solution to problem $\left(\mathrm{P}_{i}\right)$, i.e., $x_{0} \in \operatorname{LMin}\left(f_{i}, S^{i}\right)$, and by the previous remark,

$$
C_{0}\left(f_{i}\right) \cap T\left(S^{i}, x_{0}\right)=\emptyset
$$

where $C_{0}\left(f_{i}\right)=\left\{v \in \mathbb{R}^{n}: d f_{i}\left(x_{0}, v\right)<0\right\}$.

Assume that $C_{0}\left(S^{0}\right) \neq \emptyset$, then $C_{0}\left(S^{i}\right) \neq \emptyset$. By Proposition 2.6, $T\left(S^{i}, x_{0}\right)=$ $C\left(S^{i}\right)$. From (11), it follows that $C_{0}\left(f_{i}\right) \cap C_{0}\left(S^{i}\right) \subset C_{0}\left(f_{i}\right) \cap C\left(S^{i}\right)=\emptyset$. But $C_{0}\left(S^{0}\right)=C_{0}\left(f_{i}\right) \cap C_{0}\left(S^{i}\right)=\emptyset$ and we have a contradiction.

So, Global Cottle is not a true constraint qualification when some $f_{i}$ is Hadamard differentiable and (H2) holds.

(3) If there is no equality constraints, then global Cottle is not verified at a local Pareto minimum (from Prop. 2.5 and Lem. 4.1 in the next section) and, consequently, neither is (AMFCQ) in [13].

\section{Optimality CONDitions Under Generalized QUALIFICATIONS}

In this section Kuhn-Tucker type necessary optimality conditions are given for a point to be local Pareto minimum. These conditions are obtained both in primal form and in dual form, with a feasible set defined by inequality and equality constraints, the objective functions and the constraints being, at least, Dini differentiable. In order to obtain the positivity of the multipliers associated with the vector-valued objective function, a generalized constraint qualification will be assumed. In this way we generalize Maeda's results [10], which are valid for differentiable functions and without equality constraints, and Preda and Chitescu's [13] who consider a problem with Dini differentiable functions and without equality constraints. We generalize also the results of Jiménez and Novo [7], valid for differentiable problems with equality constraints.

Theorem 4.1. Let $f$ be Hadamard differentiable at $x_{0}, g_{j}, j \in J_{0}$, Dini differentiable at $x_{0}$ and $h$ Fréchet differentiable at $x_{0}$ and suppose that the generalized Abadie qualification is verified. If $x_{0} \in \operatorname{LMin}(f, S)$, then there exists no solution $v \in \mathbb{R}^{n}$ of the system

$$
\left\{\begin{array}{l}
D f\left(x_{0}, v\right) \leq 0, D f\left(x_{0}, v\right) \neq 0 \\
D g_{j}\left(x_{0}, v\right) \leq 0 \forall j \in J_{0} \\
\nabla h\left(x_{0}\right) v=0
\end{array}\right.
$$

i.e., $x_{0}$ is a proper local solution to problem (MOP) in the sense of Kuhn-Tucker. 
Proof. Assume that the conclusion is not true. Then there exist $v \in \mathbb{R}^{n}$ and $i \in\{1, \ldots, p\}$ such that

$$
\left\{\begin{array}{l}
D f_{i}\left(x_{0}, v\right)<0 \\
D f_{j}\left(x_{0}, v\right) \leq 0 \forall j \neq i \\
v \in C(S)
\end{array}\right.
$$

Thus $v \in C\left(S^{0}\right)$ and, by the generalized Abadie qualification, $v \in T\left(S^{i}, x_{0}\right)$. Since $x_{0}$ is a local Pareto minimum, it also is a local minimum of each scalar problem $\left(\mathrm{P}_{j}\right)$, in particular, $x_{0} \in \operatorname{LMin}\left(f_{i}, S^{i}\right)$. As $f_{i}$ is Hadamard differentiable, we have $D f_{i}\left(x_{0}, u\right) \geq 0 \forall u \in T\left(S^{i}, x_{0}\right)$. Taking $u=v$, then $D f_{i}\left(x_{0}, v\right) \geq 0$, in contradiction to $(13)$.

Theorem 4.2. Let $f$ and $h$ be Fréchet differentiable at $x_{0}$ and $g_{j}, j \in J_{0}$, Dini differentiable at $x_{0}$ and suppose that the generalized Guignard qualification is verified. If $x_{0} \in \operatorname{LMin}(f, S)$, then there is no solution $v \in \mathbb{R}^{n}$ of the system (12).

Proof. Assume that (13) is true for some $i \in\{1, \ldots, p\}$ and $v \in \mathbb{R}^{n}$. Thanks to the generalized Guignard qualification, $v \in \operatorname{clco} T\left(S^{i}, x_{0}\right)$. Since $x_{0} \in \operatorname{LMin}\left(f_{i}, S^{i}\right)$ and $f_{i}$ is Fréchet differentiable, we obtain $\nabla f_{i}\left(x_{0}\right) u \geq 0 \forall u \in T\left(S^{i}, x_{0}\right)$ $\left(D f_{i}\left(x_{0}, u\right)=\nabla f_{i}\left(x_{0}\right) u\right)$. By the linearity and the continuity of $\nabla f_{i}\left(x_{0}\right)(\cdot)$, it follows that $\nabla f_{i}\left(x_{0}\right) u \geq 0 \forall u \in \operatorname{cl} \operatorname{co} T\left(S^{i}, x_{0}\right)$. Taking $u=v$ we have a contradiction to (13).

It is possible to obtain the dual form of these two last theorems by applying the generalized Tucker alternative theorem (Prop. 2.2).

Theorem 4.3. Assume the hypothesis of Theorems 4.1 or 4.2 and let the derivatives $\operatorname{Df}\left(x_{0}, \cdot\right)$ and $D g_{j}\left(x_{0}, \cdot\right), j \in J_{0}$, be convex. If the cones

$D_{i}=$ cone $\operatorname{co}\left(\cup_{j \neq i} \partial_{D} f_{j}\left(x_{0}\right)\right)+\operatorname{cone} \operatorname{co}\left(\cup_{j \in J_{0}} \partial_{D} g_{j}\left(x_{0}\right)\right)+\operatorname{lin}\left\{\nabla h_{k}\left(x_{0}\right): k \in K\right\}$

$i=1, \ldots, p$, are closed, then there exists $(\lambda, \mu, \nu) \in \mathbb{R}^{p} \times \mathbb{R}^{m} \times \mathbb{R}^{r}$ such that

(a) $\lambda>0, \mu \geq 0, \mu_{j} g_{j}\left(x_{0}\right)=0, j=1, \ldots, m$;

(b) $\sum_{i=1}^{p} \lambda_{i} D f_{i}\left(x_{0}, v\right)+\sum_{j=1}^{m} \mu_{j} D g_{j}\left(x_{0}, v\right)+\sum_{k=1}^{r} \nu_{k} \nabla h_{k}\left(x_{0}\right) v \geq 0 \forall v \in \mathbb{R}^{n}$.

As usual, we take $\mu_{j}=0$ if $g_{j}\left(x_{0}\right)<0$.

Note that condition (b) is equivalent to

$$
0 \in \sum_{i=1}^{p} \lambda_{i} \partial_{D} f_{i}\left(x_{0}\right)+\sum_{j=1}^{m} \mu_{j} \partial_{D} g_{j}\left(x_{0}\right)+\sum_{k=1}^{r} \nu_{k} \nabla h_{k}\left(x_{0}\right)
$$


If we denote by $L$ the Lagrangian function: $L=\sum_{i=1}^{p} \lambda_{i} f_{i}+\sum_{j=1}^{m} \mu_{j} g_{j}+$ $\sum_{k=1}^{r} \nu_{k} h_{k}$, then (16) is equivalent to

$$
0 \in \partial_{D} L\left(x_{0}\right)
$$

Theorem 4.3 generalizes Corollary 8 in [7] by Jiménez and Novo.

Now we investigate about the conditions on the functions of the problem, which assume that the cones (14) are closed. One of these criteria is given below.

Proposition 4.1. If for each $i=1, \ldots, p, C_{0}\left(S^{i}\right) \neq \emptyset$, then the cones $D_{i}, i=$ $1, \ldots, p$, given by (14), are closed.

This follows from Proposition 3.6 in [8].

We remark that if the Cottle qualification holds, then it is unnecessary to use the generalized Tucker alternative theorem to obtain positive multipliers, since this result can directly be obtained.

Proposition 4.2. Let $f$ be Hadamard differentiable at $x_{0}$ with convex derivative and suppose that the Cottle qualification and (H2) are satisfied. If $x_{0} \in \operatorname{LMin}(f, S)$, then conditions (15) hold.

Proof. From Theorem 3.1, part 6, the generalized Abadie qualification is verified and, by Theorem 4.1, the system (12) does not admit solution.

For each $i=1, \ldots, p$ we have $x_{0} \in \operatorname{LMin}\left(f_{i}, S^{i}\right)$, hence $D f_{i}\left(x_{0}, v\right) \geq 0 \forall v \in$ $T\left(S^{i}, x_{0}\right)$. As it was seen in the proof of Theorem 3.1, part 6 , for each $i=1, \ldots, p$, $T\left(S^{i}, x_{0}\right)=C\left(S^{i}\right)$. Therefore, none of the $p$ systems $(i=1, \ldots, p)$ :

$$
\left\{\begin{array}{l}
D f_{i}\left(x_{0}, v\right)<0 \\
D f_{j}\left(x_{0}, v\right) \leq 0 \forall j \neq i \\
D g_{j}\left(x_{0}, v\right) \leq 0 \forall j \in J_{0} \\
\nabla h_{k}\left(x_{0}\right) v=0 \forall k \in K
\end{array}\right.
$$

has a solution $v \in \mathbb{R}^{n}$. Let us consider the convex problem

$$
\begin{aligned}
\left(\mathrm{CP}_{i}\right) \alpha_{i}=\operatorname{Min}\left\{D f_{i}\left(x_{0}, v\right):\right. & D f_{j}\left(x_{0}, v\right) \leq 0 \forall j \neq i, \\
D g_{j}\left(x_{0}, v\right) & \left.\leq 0 \forall j \in J_{0}, \quad \nabla h_{k}\left(x_{0}\right) v=0 \forall k \in K\right\} .
\end{aligned}
$$

Because of the incompatibility of the system (18) above, we have $\alpha_{i} \geq 0$. Since $v=0$ is a feasible solution and $D f_{i}\left(x_{0}, 0\right)=0$, it is $\alpha_{i}=0$. From Theorem 28.2 in [14] (we can use it because $C_{0}\left(S^{i}\right) \neq \emptyset$ ), it follows that there exist $\lambda_{i j} \geq 0$, $j \neq i ; \mu_{i j} \geq 0, j \in J_{0} ; \nu_{i k} \in \mathbb{R}, k \in K$ such that

$$
D f_{i}\left(x_{0}, v\right)+\sum_{j=1, j \neq i}^{p} \lambda_{i j} D f_{j}\left(x_{0}, v\right)+\sum_{j \in J_{0}} \mu_{i j} D g_{j}\left(x_{0}, v\right)+\sum_{k=1}^{r} \nu_{i k} \nabla h_{k}\left(x_{0}\right) v \geq 0
$$


for all $v \in \mathbb{R}^{n}$, and for $i=1, \ldots, p$. Adding over $i=1, \ldots, p$, we have

$$
\sum_{i=1}^{p} \lambda_{i} D f_{i}\left(x_{0}, v\right)+\sum_{j \in J_{0}} \mu_{j} D g_{j}\left(x_{0}, v\right)+\sum_{k=1}^{r} \nu_{k} \nabla h_{k}\left(x_{0}\right) v \geq 0 \forall v \in \mathbb{R}^{n}
$$

where, in order to simplify, we have denoted $\lambda_{i}=1+\sum_{j=1, j \neq i}^{p} \lambda_{j i}, i=1, \ldots, p$; $\mu_{j}=\sum_{i=1}^{p} \mu_{i j}, j \in J_{0} ; \nu_{k}=\sum_{i=1}^{p} \nu_{i k}, k=1, \ldots, r$, and obviously we have $\lambda>0$, and $\mu \geq 0$.

As a consequence of Theorem 4.3 we obtain the following corollary, which extends Maeda's Theorem 3.2 [10] for a problem with differentiable functions and also equality constraints.

Corollary 4.1 ([7], (Cor. 8)). Let $f, g$ and $h$ be Fréchet differentiable at $x_{0}$ and suppose that the generalized Guignard qualification is verified. If $x_{0} \in \operatorname{LMin}(f, S)$, then there exist $(\lambda, \mu, \nu) \in \mathbb{R}^{p} \times \mathbb{R}^{m} \times \mathbb{R}^{r}$ such that

(a) $\lambda>0, \mu \geq 0, \mu_{j} g\left(x_{0}\right)=0, j=1, \ldots, m$;

(b) $\sum_{i=1}^{p} \lambda_{i} \nabla f_{i}\left(x_{0}\right)+\sum_{j=1}^{m} \mu_{j} \nabla g_{j}\left(x_{0}\right)+\sum_{k=1}^{r} \nu_{k} \nabla h_{k}\left(x_{0}\right)=0$.

Proof. Under these assumptions, the condition "for each $i=1, \ldots, p, D_{i}$ is closed" is verified, since

$$
D_{i}=\operatorname{coneco}\left(\left\{\nabla f_{j}\left(x_{0}\right): j \neq i\right\} \cup\left\{\nabla g_{j}\left(x_{0}\right): j \in J_{0}\right\}\right)+\operatorname{lin}\left\{\nabla h_{k}\left(x_{0}\right): k \in K\right\}
$$

is a polyhedral convex cone and, therefore, it is closed.

The following example shows that Cottle qualification may not be verified; however, we can apply Theorem 4.3.

Example 4.1. In $\mathbb{R}^{3}$, let $x_{0}=(0,0,0), f_{1}=2 x-2 z, f_{2}=-2 y$ and let $g$ be the support function of the set $B=\left\{(x, y, z): x^{2}+(y-2)^{2}+z^{2} \leq 2, z \geq 0\right\}$. We obtain the following expression of $g$ :

$$
g(x, y, z)=\left\{\begin{array}{lll}
2 y+\sqrt{2 x^{2}+2 y^{2}+2 z^{2}} & \text { if } \quad z \geq 0 \\
2 y+\sqrt{2 x^{2}+2 y^{2}} & \text { if } \quad z<0 .
\end{array}\right.
$$

Obviously $D g\left(x_{0}, v\right)=g(v)$ and $\partial_{D} g\left(x_{0}\right)=B$. The feasible set is $G=\{(x, y, z)$ : $g(x, y, z) \leq 0\}$ and the point $x_{0}$ is a Pareto minimum of $f=\left(f_{1}, f_{2}\right)$ over $G$. Cottle qualification is not verified because $C_{0}\left(S^{1}\right)=\left\{v: \nabla f_{2}\left(x_{0}\right) v<0, D g\left(x_{0}, v\right)<\right.$ $0\}=\emptyset$, but we can apply Theorem 4.3 , since the cones $D_{1}=\operatorname{coneco}(\{(0,-2,0)\} \cup$ $B)=\{(x, y, z): \quad z \geq 0\}$ and $D_{2}=\operatorname{coneco}(\{(2,0,-2)\} \cup B)$ are closed $\left(D_{2}\right.$ is closed because $C_{0}\left(S^{2}\right) \neq \emptyset$, since $\left.(-1,-2,0) \in C_{0}\left(S^{2}\right)\right)$. So (16) holds, with $\left(\lambda_{1}, \lambda_{2}, \mu\right)=(1,2,2)$ and $b=(-1,2,1) \in \partial_{D} g\left(x_{0}\right)$.

Finally, we establish necessary optimality conditions without equality constraints; moreover, we do not require the objective functions to be Hadamard differentiable as in Theorems 4.1 and 4.2 . 
In Lemma 4.1 and Theorem 4.4 the following definition is used: $\varphi: \mathbb{R}^{n} \rightarrow \mathbb{R}$ is quasiconvex at $x_{0}$ on a neighborhood of $x_{0}$ if there exists $\delta>0$ such that

$$
x \in B\left(x_{0}, \delta\right), \varphi(x) \leq \varphi\left(x_{0}\right) \Rightarrow \varphi\left(\lambda x_{0}+(1-\lambda) x\right) \leq \varphi\left(x_{0}\right) \forall \lambda \in(0,1) .
$$

Lemma 4.1. If $f$ has continuous Dini derivative at $x_{0}, g_{j}, j \in J_{0}$, are quasiconvex at $x_{0}$ on a neighborhood of $x_{0}$ and $x_{0} \in \operatorname{LWMin}(f, G)$, then

$$
T\left(G, x_{0}\right) \cap C_{0}(F)=\emptyset .
$$

Proof. Assume that $T\left(G, x_{0}\right) \cap C_{0}(F) \neq \emptyset$ and choose $v \in T\left(G, x_{0}\right) \cap C_{0}(F)$. Thus, $\operatorname{Df}\left(x_{0}, v\right)<0$. Using Lemma 4.10 in $[8], \operatorname{cl} Z\left(G, x_{0}\right)=T\left(G, x_{0}\right)$. Hence there exist $v_{n} \in Z\left(G, x_{0}\right)$ such that $v_{n} \rightarrow v$. By the continuity of $D f\left(x_{0}, \cdot\right)$, we can suppose that $D f\left(x_{0}, v_{n}\right)<0 \forall n \in \mathbb{N}$. By the definition of Dini derivative, $D f\left(x_{0}, v_{n}\right)=$ $\lim _{t \rightarrow 0^{+}}\left(f\left(x_{0}+t v_{n}\right)-f\left(x_{0}\right)\right) / t<0$. Therefore, there exists $\delta_{n}>0$ such that $\forall t \in\left(0, \delta_{n}\right], f\left(x_{0}+t v_{n}\right)-f\left(x_{0}\right)<0$. As $v_{n} \in Z\left(G, x_{0}\right)$, there exists $\eta_{n}>0$ such that $\forall t \in\left(0, \eta_{n}\right], x_{0}+t v_{n} \in G$. Let us choose $\varepsilon_{n}$ such that $0<\varepsilon_{n} \leq \operatorname{Min}\left\{\delta_{n}, \eta_{n}\right\}$ and $\varepsilon_{n} \rightarrow 0^{+}$. The sequence $x_{n}=x_{0}+\varepsilon_{n} v_{n} \rightarrow x_{0}, x_{n} \in G$ and $f\left(x_{n}\right)<f\left(x_{0}\right)$, in contradiction with the minimality of $x_{0}$.

Theorem 4.4. Let $\mathrm{S}=\mathrm{G}$ and assume that:

(a) $f_{i}, i \in I, g_{j}, j \in J_{0}$, are quasiconvex at $x_{0}$ on a neighborhood of $x_{0}$.

(b) $f_{i}, i \in I, g_{j}, j \in J_{0}$, are Dini differentiable at $x_{0}$, with $D f_{i}\left(x_{0}, \cdot\right), i \in I$, linear and $D g_{j}\left(x_{0}, \cdot\right), j \in J_{0}$, convex.

(c) The generalized Guignard qualification holds.

If $x_{0} \in \operatorname{LMin}(f, G)$, then the system

$$
\left\{\begin{array}{l}
D f\left(x_{0}, v\right) \leq 0, D f\left(x_{0}, v\right) \neq 0 \\
D g_{j}\left(x_{0}, v\right) \leq 0 \forall j \in J_{0}
\end{array}\right.
$$

has no solution $v \in \mathbb{R}^{n}$.

Proof. Assume the thesis does not hold. Then there exist $v \in \mathbb{R}^{n}$ and $i \in\{1, \ldots, p\}$ such that

$$
\left\{\begin{array}{l}
D f_{i}\left(x_{0}, v\right)<0 \\
D f_{j}\left(x_{0}, v\right) \leq 0 \forall j \neq i \\
D g_{j}\left(x_{0}, v\right) \leq 0 \forall j \in J_{0} .
\end{array}\right.
$$

Therefore $v \in C\left(S^{0}\right)$ and, using condition (c), $v \in \cap_{j=1}^{p} \operatorname{clco} T\left(S^{j}, x_{0}\right)$. Consequently, $v \in \operatorname{clco} T\left(S^{i}, x_{0}\right)$. Since $x_{0} \in \operatorname{LMin}(f, S)$, it follows that $x_{0} \in$ $\operatorname{LMin}\left(f_{i}, S^{i}\right)$. Since $D f_{i}\left(x_{0}, \cdot\right)$ is continuous (it is linear) and as hypothesis (a) holds, we can apply Lemma 4.1, obtaining $D f_{i}\left(x_{0}, u\right) \geq 0 \forall u \in T\left(S^{i}, x_{0}\right)$. Moreover, by the linearity, we deduce that $D f_{i}\left(x_{0}, u\right) \geq 0 \forall u \in \operatorname{cl} \operatorname{co} T\left(S^{i}, x_{0}\right)$. In particular, taking $u=v$, we obtain $D f_{i}\left(x_{0}, v\right) \geq 0$, contradicting (19).

This theorem improves Theorem 3.1 in Preda and Chitescu [13] because quasiconcavity of $f_{i}$ is not required. 
The following theorem can be proved in a similar. From this result, we obtain subsequently (Th. 4.6) necessary optimality conditions in dual form.

Theorem 4.5. Assume the hypotheses of Theorem 4.4, with $D f_{i}\left(x_{0}, \cdot\right), i \in I$, convex (instead of linear) and

$\left(c^{\prime}\right)$ The generalized Abadie qualification holds,

(instead of $(c)$ ). Then the same conclusion of Theorem 4.4 holds.

Theorem 4.6. Suppose that the hypotheses of Theorems 4.4 or 4.5 hold true. If for each $i=1, \ldots, p$ the cone

$$
D_{i}=\operatorname{coneco}\left(\cup_{j \neq i} \partial_{D} f_{j}\left(x_{0}\right)\right)+\operatorname{coneco}\left(\cup_{j \in J_{0}} \partial_{D} g_{j}\left(x_{0}\right)\right)
$$

is closed, then there exists $(\lambda, \mu) \in \mathbb{R}^{p} \times \mathbb{R}^{m}$ such that

(a) $\lambda>0, \mu \geq 0, \mu_{j} g_{j}\left(x_{0}\right)=0, j=1, \ldots, m$;

(b) $\sum_{i=1}^{p} \lambda_{i} D f_{i}\left(x_{0}, v\right)+\sum_{j=1}^{m} \mu_{j} D g_{j}\left(x_{0}, v\right) \geq 0 \quad \forall v \in \mathbb{R}^{n}$.

Expressions similar to equations (16) and (17) can also be obtained for (b).

This theorem corrects Theorem 3.2 in Preda and Chitescu [13], which is not true, as the following counterexample shows.

Example 4.2. Let us consider the problem

$$
\text { Min } f(x) \text { subject to } g(x) \leq 0,
$$

where $f: \mathbb{R}^{3} \rightarrow \mathbb{R}^{2}$ is given by $f(x, y, z)=(x,-y)$ and $g(x, y, z)=y+z+$ $\sqrt{x^{2}+y^{2}+z^{2}}$. We are going to study the minimality conditions at the point $x_{0}=(0,0,0)$.

The feasible set $S=\{(x, y, z): g \leq 0\}$ is the regular cone (in the sense of elementary geometry) with axis the halfline $(x, y, z)=\lambda(0,-1,-1), \lambda \geq 0$ and whose generatrices form an angle of $\pi / 4$ with the axis of the cone. We have

$$
\begin{aligned}
S^{1} & =\left\{(x, y, z): f_{2} \leq 0, g \leq 0\right\}=\{(0,0, z): z \leq 0\}, \\
S^{2} & =\left\{(x, y, z): f_{1} \leq 0, g \leq 0\right\}=S \cap\{x \leq 0\}, \\
S^{0} & =\left\{(x, y, z): f_{1} \leq 0, f_{2} \leq 0, g \leq 0\right\}=S^{1}, \\
\nabla f_{1}\left(x_{0}\right) & =(1,0,0), \nabla f_{2}\left(x_{0}\right)=(0,-1,0), D g\left(x_{0}, v\right)=g(v) .
\end{aligned}
$$

We are under the hypotheses of Theorem 3.2 in Preda and Chitescu [13]:

1. The point $x_{0}$ is a Pareto minimum, since $f\left(x_{0}\right)=(0,0)$ and if $(x, y, z) \in S$, then $f(x, y, z)-f\left(x_{0}\right) \in-\mathbb{R}_{+}^{2} \backslash\{0\}$ is not true (note that $S$ is inside the dihedral $\{(x, y, z): y \leq 0, z \leq 0\}$ and it cuts the plane $y=0$ only in the halfline $\left.O Z^{-}\right)$. 2. The generalized Guignard qualification is true, because $\operatorname{clco} T\left(S^{1}, x_{0}\right) \cap \operatorname{cl} \operatorname{co} T$ $\left(S^{2}, x_{0}\right)=S^{1}$ and $C\left(S^{0}\right)=S^{0}=S^{1}$.

3. $f_{1}, f_{2}$ are linear, and consequently they are quasiconvex and quasiconcave; $g$ is convex, and therefore, it also is quasiconvex.

4. $f_{1}, f_{2}$ are Fréchet differentiable, thus their Dini derivatives are linear, and hence, concave and convex. $g$ is Hadamard differentiable and its derivative at $x_{0}$ is $g$ itself, which is a convex function. 
However, there exist no $\lambda_{1}>0, \lambda_{2}>0, \mu \geq 0$ such that

$$
\lambda_{1} \nabla f_{1}\left(x_{0}\right) v+\lambda_{2} \nabla f_{2}\left(x_{0}\right) v+\mu D g\left(x_{0}, v\right) \geq 0 \quad \forall v \in \mathbb{R}^{3} .
$$

In fact, the unique solution with $\mu=0$ is $\lambda_{1}=\lambda_{2}=0$. Then, let $\mu>0$; we can assume $\mu=1$ and put $v=(x, y, z)$. Then (20) is equivalent to

$$
\left\langle\left(-\lambda_{1}, \lambda_{2}-1,-1\right),(x, y, z)\right\rangle \leq\|(x, y, z)\| \quad \forall(x, y, z) \in \mathbb{R}^{3} .
$$

This expression means that $\left(-\lambda_{1}, \lambda_{2}-1,-1\right)$ is a subgradient at 0 of the convex function $x \mapsto\|x\|$. But $\partial\|\cdot\|(0)=\operatorname{cl} B(0,1)$ and therefore $\lambda_{1}^{2}+\left(\lambda_{2}-1\right)^{2}+1 \leq 1$, and the only solution is $\left(\lambda_{1}, \lambda_{2}\right)=(0,1)$.

Note that Theorem 4.6 cannot be applied, because the cone

$$
D_{1}=\operatorname{cone}\left\{\nabla f_{2}\left(x_{0}\right)\right\}+\text { cone } \partial_{D} g\left(x_{0}\right)=\{(x, y, z): z>0 \text { or }(z=0, x=0)\}
$$

is not closed, being $\partial_{D} g\left(x_{0}\right)=\operatorname{cl} B\left(b_{0}, 1\right)$ with $b_{0}=(0,1,1)$.

\section{REFERENCES}

[1] J.P. Aubin and H. Frankowska, Set-valued analysis. Birkhaüser, Boston (1990).

[2] M.S. Bazaraa and C.M. Shetty, Foundations of optimization. Springer-Verlag, Berlin (1976).

[3] M.S. Bazaraa and C.M. Shetty, Nonlinear programming. John Wiley \& Sons, New York (1979).

[4] V.F. Demyanov and A.M. Rubinov, Constructive nonsmooth analysis. Verlag Peter Lang, Frankfurt am Main (1995).

[5] G. Giorgi and S. Komlósi, Dini derivatives in optimization. Part I. Riv. Mat. Sci. Econom. Social. Anno 15 (1992) 3-30.

[6] Y. Ishizuka, Optimality conditions for directionally differentiable multiobjective programming problems. J. Optim. Theory Appl. 72 (1992) 91-111.

[7] B. Jiménez and V. Novo, Cualificaciones de restricciones en problemas de optimización vectorial diferenciables. Actas XVI C.E.D.Y.A./VI C.M.A. Vol. I, Universidad de Las Palmas de Gran Canaria, Spain (1999) 727-734.

[8] B. Jiménez and V. Novo, Alternative theorems and necessary optimality conditions for directionally differentiable multiobjective programs. J. Convex Anal. 9 (2002) 97-116.

[9] B. Jiménez and V. Novo, Optimality conditions in directionally differentiable Pareto problems with a set constraint via tangent cones. Numer. Funct. Anal. Optim. 24 (2003) 557-574.

[10] T. Maeda, Constraint qualifications in multiobjective optimization problems: differentiable case. J. Optim. Theory Appl. 80 (1994) 483-500.

[11] O.L. Mangasarian, Nonlinear programming. McGraw-Hill, New York (1969).

[12] V. Novo and B. Jiménez, Lagrange multipliers in multiobjective optimization under mixed assumptions of Fréchet and directional differentiability, in 5th International Conference on Operations Research, University of La Habana, Cuba, March 4-8 (2002). Investigación Operacional 25 (2004) 34-47.

[13] V. Preda and I. Chitescu, On constraint qualification in multiobjective optimization problems: semidifferentiable case. J. Optim. Theory Appl. 100 (1999) 417-433.

[14] R.T. Rockafellar, Convex Analysis. Princeton University Press, Princeton (1970). 